\title{
CONCEPTS FOR HYBRID MICRO ASSEMBLY USING HOT MELT JOINING
}

\author{
Sven Rathmann, Annika Raatz, Jürgen Hesselbach
}

Institute of Machine Tools and Production Engineering

Technical University Braunschweig

Langer Kamp 19b, 38106 Braunschweig, Germany

\begin{abstract}
Nowadays, the production of 3D MEMS and MOEMS is carried out by using hybrid integration of single components, for which batch production is normally preferred. In this field, adhesive technology is one of the major joining techniques. At the Collaboration Research Center 516, a batch process based on a joining technique which uses hot melt adhesives was developed. This technique allows the coating of micro components with hot melt in a batch. The coating process is followed by the joining process. Due to this, the time between coating and joining can be designed variably. Because of the short set times of hot melt adhesives, short joining times are possible. For this assembly process adapted heat management is necessary. This paper presents adapted heating management concepts and gripping systems which allow a fast and accurate assembly of hybrid micro systems with hot melt coated components. Therefore, the chosen gripping system depends on the process and heat management concept as well as the thermal properties of the components. Furthermore, the simulative and experimental results of the heat management concepts will be discussed.
\end{abstract}

Keywords precision joining operations, hot melt adhesives, heat management concept

\section{Introduction}

The continuous miniaturisation of hybrid micro systems requires joining processes which are suitable for batch production [1]. The most important technology is the adhesive joining technique [2]. Mostly, viscous adhesives are used. Disadvantages of viscous adhesives are long set times and the low suitability for batch processes. A new approach is the usage of hot melt adhesives. The main advantages are extremely short set cycles, the possibility of pre-applying the adhesive and the timedelayed joining procedure [3]. Therefore, the use of hot melt adhesives can be an interesting alternative for the assembly of hybrid micro systems. To establish this technology, adhesive and application technologies as well as the assembly process

Please use the following format when citing this chapter.

Rathmann, S., Raatz, A., Hesselbach, J., 2008, in IFIP International Federation for Information Processing, Volume 260, Micro-Assembly Technologies and Applications, eds. Ratchev, S., Koelemeijer, S., (Boston: Springer), pp. 161-169. 
must be developed, and the process parameters must be determined. A very important aspect for the process design is the kind of heating technology. Therefore, special process components, such as fixtures and grippers as well as the heat management concepts should be developed. One project at the Collaborative Research Centre 516 "Design and Manufacturing of Active Microsystems" is concerned with the development and modification of hot melt adhesives and coating concepts and also with the development of the special assembly process with hot melt coated micro components.

\section{Joining Technology}

The listed limitations of viscous adhesives in the micro system engineering are to be compensated by using hot melts. These hot melts are thermoplastics, physical setting adhesives, which are single-component, non-viscous and non-solvent at room temperature. One of the most important advantages of hot melts in comparison to viscous adhesive systems is the possibility of pre-applying hot melt systems in a batch process, e.g. as powder or adhesive spheres, as dispersion or as an adhesive foil. The joining procedure does not have to take place directly after the adhesive has been applied to the substrate; this can happen at any time later on, i.e. hot melts possess no pot life time [4]. The adhesive is only melted during the bonding process by a thermal impulse and moistens the surface of the other substrate. The heating can be accomplished directly by heating the adhesive itself or indirectly by heating the substrate. The adhesive sets, once the temperature of the adhesive has fallen below the melting temperature. If an appropriate heat management concept is used, the hot melts set very fast, which means that the handling strength (usually the ultimate strength) can be achieved in less than one second as experiments have shown.

\section{Assembly Process}

This section discusses the design of an assembly process which makes use of a joining technique based on the application of hot melt adhesives described above. The design of the joining process is the main focus when developing the assembly process [5]. However, in order to put the concept into practice, further requirements have to be imposed with regard to the design of the process. First, the clamping and gripping units should not come into contact with the adhesive covered surfaces of the components, once the hot melt has been melted. Second, the coating should not distort or cover any marks and parts of the component needed for the assembly. Third, possible ways of how the necessary heat can be transmitted into the components must be considered, which is the most important of all requirements. The different heat management concepts which are usable will be discussed on the basis of a specific micro system, which is presented below. Figure 1 shows the assembly 
scenario of a linear micro stepping motor. This motor consists of a stator, two guide blocks, a traveller, a traveller block as well as guiding balls. Moreover, a distance foil is needed for the assembly.

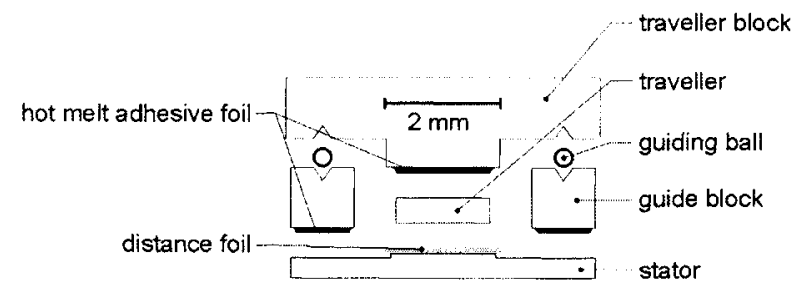

Fig. 1. Example product for micro assembly using hot melt adhesives

The assembly steps are: joining the guide blocks, inserting the distance foil, placing the traveller, inserting the guiding balls and joining the traveller block with the traveller. In a pre-assembly step, a thin film of hot melt adhesive (about $50 \mu \mathrm{m}$ ) is applied onto the guide blocks and the traveller block. The melting temperature of the hot melt ranges from $100^{\circ} \mathrm{C}$ to $110^{\circ} \mathrm{C}$. In the following, the assembly process will be discussed on the basis of the joining procedure of a guide block, which consists of six steps:

1. measuring the position of the stator

2. measuring the position of the guide block

3. gripping the guide block

4. heating up the hot melt coated guide block

5. aligning the guide block with the joining position

6. joining the guide block with the stator

The heating of the hot melt adhesive can be carried out in various ways. Not only does the kind of heat source play an important role for the process design, but also the way the heat should be transmitted into the component as well as the starting time of the heat input must be taken into account. These factors exert influence also on the load of the components during the heating phase and the assembly uncertainty. In the next two sections, the different heat management concepts and experimental results will be presented and their advantages and disadvantages analyzed.

\section{Adaptive Heat Management Concepts}

Heat management is an integral part for the selection and the design of an assembly process using hot melt adhesives. Since the volume of the hot melt is quite small, the thermal capacity is rather low, which is why heating the hot melt itself is not very practical. Contrarily, the thermal capacity of the component and the gripping system is much higher. Hence, the characteristics of the grippers and the components such as their volume, their thermal capacity as well as their thermal conduc- 
tivity must be taken into consideration when designing the assembly process. Besides, the heating source is another crucial factor for the process design. The following heat sources can be used to heat up the gripping unit: heating plates, infrared heaters, lasers, heating foils and Peltier elements as well as a combination of these heat sources. In general, there are two different kinds of heat management concepts - a passive and an active one. The passive heat management concept makes use of the principle of heat storage to supply the energy for the joining process.

The solid line in Figure 2 shows the typical temperature history of a component according to the passive heat management concept. Before the actual joining process, the gripper and the component inside it are heated up by a heat source until the working temperature $T_{H p}$ has been reached. The time needed until $T_{H p}$ has been reached determines the process time to a large extent. If neither handling nor measuring operations have to take place right after the gripper has picked up the component, the gripper should be heated up before the gripping process. Besides, the working temperature $T_{V}$ must be much higher than the melting temperature of the adhesive to provide enough time to position and join the components until the temperature falls below the working temperature, which is marked by $t_{K p}$. The time span between $t_{\mathrm{Hp}}$ and $t_{\mathrm{Kp}}$ is referred to as the processing time. Contrary to the setting, the process time marks the time span during which the position of the components can still be measured and adjusted. The processing time depends mostly on the material of the components and the gripper. Once the joining component comes into contact with its joining partner, the temperature drops substantially, whereupon the hot melt sets and reaches its final strength. In general, the assembly time is less than a second.

As Figure 2 shows, the working temperature is much higher than the melting temperature, as a result of which the components are exposed to very high thermal load. Under certain circumstances, this load will lead to a deterioration of the accuracy of the assembly process. Nonetheless, the fact that the passive heat management concept can be easily integrated into already existing assembly systems is a major advantage of this concept. Disadvantages are the long heating times, the inflexibility of the process design, the high thermal load of the components as well as the immense efforts to monitor the assembly process. In particular, the controlling process of the temperature variation is rather complex.

In contrast to the passive heat management concept, the heat source of the active heat management concept is integrated into the gripping or clamping unit. Therefore, heat can be continuously transmitted into the component during the handling process. As another result, the temperature- and material-dependent processing time of the passive heat management concept can be omitted, which is why the processing temperature can be set at a lower level. In most cases the processing temperature can be set right above the melting point. This results in a lower thermal load of the components. Due to the continuous heat input, the processing time of the active heat management concept can be variably chosen. Thus, the assembly process can be designed very flexibly. By actively cooling the components, the joining time can be further shortened. The typical temperature profile of the active heat management concept is illustrated by the dashed line in Figure 2. 


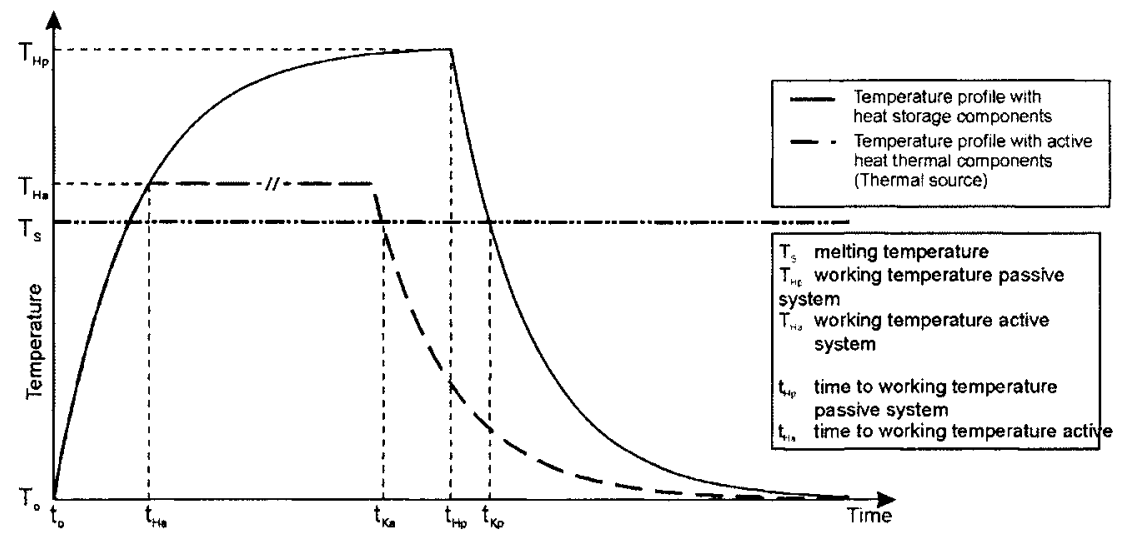

Fig. 2. Temperature profiles on different heat managements

The advantage of the active heat management concept is the independence of the assembly process from the heat capacity of components which leads to a flexible process design. The complex design and the integration of active heating components is a disadvantage of the active management concept, mainly, if small and accurate assembly systems are used. In contrast, the integration of heat storage components is easier to handle. However, the control and monitoring of the passive concept, especially the control and monitoring of the temperature, are considerably more difficult than with the active heat management concept. The described heat management concepts allow a variable design of the assembly processes. However, not only the active or the passive heat management concept can be used, but also a combination of both is possible.

\section{Experimental Setup}

For the experimental validation, a micro assembly system was used. Figure 3 shows the micro assembly robot AUTOPLACE 411 with different fixture and heating systems. The robot has 4 DOF. The uncertainty of the robot system is $1 \mu \mathrm{m}$ in the $x-$, $y$-, and z-direction. An adapted gripper system is mounted onto the robot system. For the several heating concepts, a precision heating plate and an infra red heater are used. The IR heater has a surface power of $64 \mathrm{~kW} / \mathrm{m}^{2}$ with a typical working temperature of $860^{\circ} \mathrm{C}$.

To investigate the passive and the active heat management concepts a passive and an active gripper system were designed. Figure 4 shows the passive gripper concept for the assembly of the guide blocks in Figure 1. It consists of a base plate, isolation ceramic and a vacuum gripper with an integrated heat accumulator. The material of the heat accumulator is copper. The heating of the guide block and the gripper is done by an infra red heater. The emitted wave length ranges from 2 to $10 \mu \mathrm{m}$. Due to the high reflection and transmission index, the heat input in the guide block into makes up only $6 \%$ of the emitted heat radiation [6]. Hence, the 
guide block is heated by conduction between the gripper system and the guide block. The heat that is needed for this process is brought into the gripping system through heat radiation.

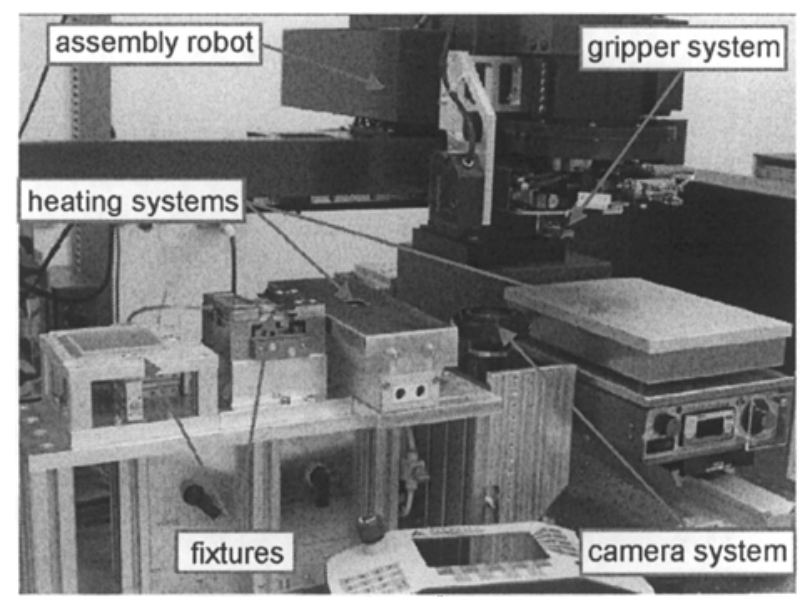

Fig. 3. Assembly robot AUTOPLACE 411 (SYSMELEC)

Figure 5 shows an active gripper concept. The heat source of the gripper is a peltier element. The gripper consists of a heat sink, isolation between heat sink and the heat flux elements and the peltier element in the middle of the active gripper. The gripping concept has to guarantee a good contact surface for the heat flux from the gripper to the guide block.

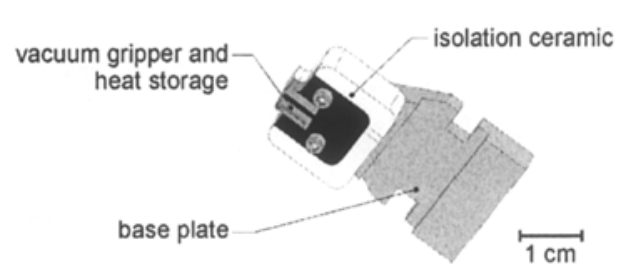

Fig. 4. Passive gripper concept

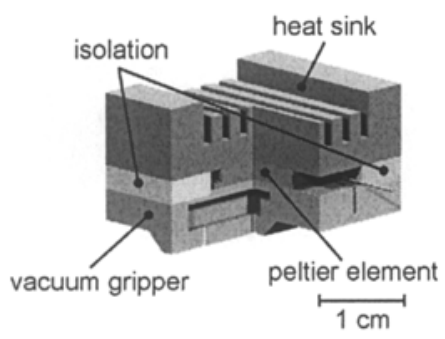

Fig. 5. Active gripper concept 


\section{Simulation and Experimental Results}

As a first step the process parameters have to be defined. Parameters of interest are: the warming time, the cooling time, the components' temperature and the heat flux to the ambiance. A simulation model was designed to determine these parameters. Figure 6 shows the passive gripper simulation model and the result at the time of $50 \mathrm{~s}$ of a transient temperature simulation. The gripper system is heated with an infra red heater. The infra red radiation is simulated by a heat flux to the gripper system. For the calculation of the heat flux an effective area of $144 \mathrm{~mm}^{2}$ was assumed. Based on the heater properties, the heat flux to the gripper system, which affects to the bottom of it, was estimated at $9 \mathrm{~W}$.

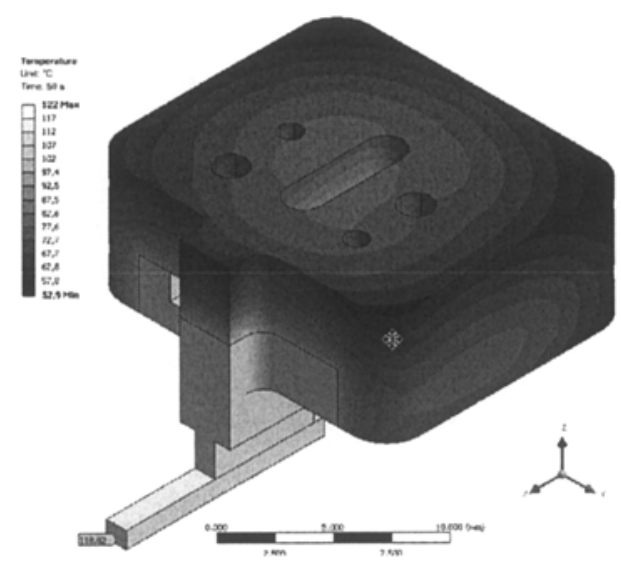

Fig. 6. Simulation model and results

The transient simulation shows the heat distribution in the gripper and the picked guiding block during and after the warming phase of $50 \mathrm{~s}$ (Figure 7). The maximum gripper temperature is $122^{\circ} \mathrm{C}$. The hot melt coated area of the guide block, the bottom side, has a mean temperature $\mathrm{T}_{\mathrm{Hp}}$ of $121^{\circ} \mathrm{C}$ at this time. 


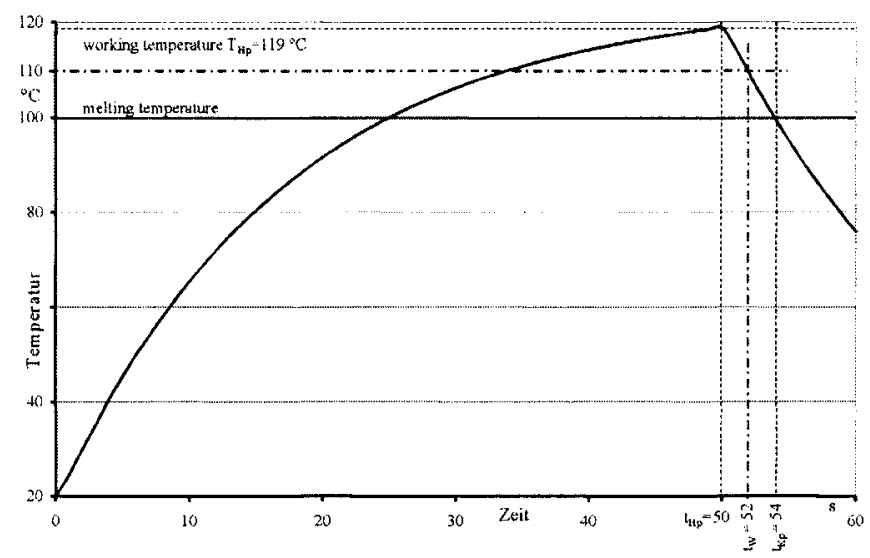

Fig. 7. Temperature profile of the hot melt coated side of the guide block

For the movement to the assembly position a maximum time of $2 \mathrm{~s}$ is scheduled. After this time the mean temperature on the coated side of the guide block is $110^{\circ} \mathrm{C}$, which is adequate for the hot melt joining process. The critical time $t_{\mathrm{Kp}}$, i.e. the time that elapses until the temperature has fallen right below the melting point, is reached $4 \mathrm{~s}$ after the warming phase. To control and validate the simulation parameters and results, the passive heat management concept was implemented for the described micro assembly system. As the temperature is difficult to measure, only samples of the gripper temperature can be taken. But the results from the simulation are in accordance with the results of the experiments that were carried out.

\section{Conclusion and Outlook}

In this paper an alternative joining process for hybrid micro systems based on hot melt adhesives was presented. In particular, the process design of the joining process in combination with different heat management concepts was described. In this context, an active and a passive heat management concept were discussed. The objective of this work is to determine the expedient parameters to design the assembly process. As a first step, the simulation results and the experimental validation show that the passive heat management concept is suitable for joining with hot melt adhesives. The disadvantage of the passive management concept, i.e. the long heating time, can be reduced by using the active concept. The first steps for an active heat management concept have been done by developing an active gripper system. Next steps will be the integration of the assembly system and experimental investigations. In the active management concept has to be investigated. In addition, the combination of the active and passive heat management concept seems to be expedient for a joining technology in micro assembly using hot melt adhesives. 


\section{Acknowledgments}

The authors gratefully acknowledge the funding of the reported work by the German Research Centre (Collaborative Research Centre 516 "Design and Manufacturing of Active Micro Systems")

\section{References}

1. H. Van Brussel, et. Al., Assembly of micro systems. In: Annals of the CIRP, 49(2), pp. 451-472 (2000).

2. M.F. Zäh, M. Schilp and D. Jacob, Kapsel und Tropfen - Fluidauftrag für Mikrosysteme. Evolutionäre und revolutionäre Verfahren in der Dispenstechnik. In: WtWerkstattstechnik online 92(9), pp. 428-431 (2002).

3. S. Böhm, et Al., Micro Bonding with non-viscous adhesives. In: Microsystem Technologies 12(7), pp. 676-679 (2006).

4. S. Böhm, et Al., Micro Bonding using hot melt adhesives. Journal of Adhesion and Interface. The Society of Adhesion \& Interface 7(4), pp. 28-31 (2006).

5. B. Lotter and H.-P.Wiendahl, (editors), Montage in der indusriellen Produktion - Ein Handbuch für die Praxis. Springer Verlag Berlin Heidelberg, ISBN 3-540-21413-5 (2006).

6. R. Hull, Properties of Crystalline Silicon. Emis DataReviews Series No. 20, INSPEC, IEE, London, UK (1999). 